\title{
Determination of location of Top Dead Centre and compression ratio value on the basis of ship engine indicator diagram
}

\author{
Stanisław Polanowski, Prof. \\ Polish Naval University
}

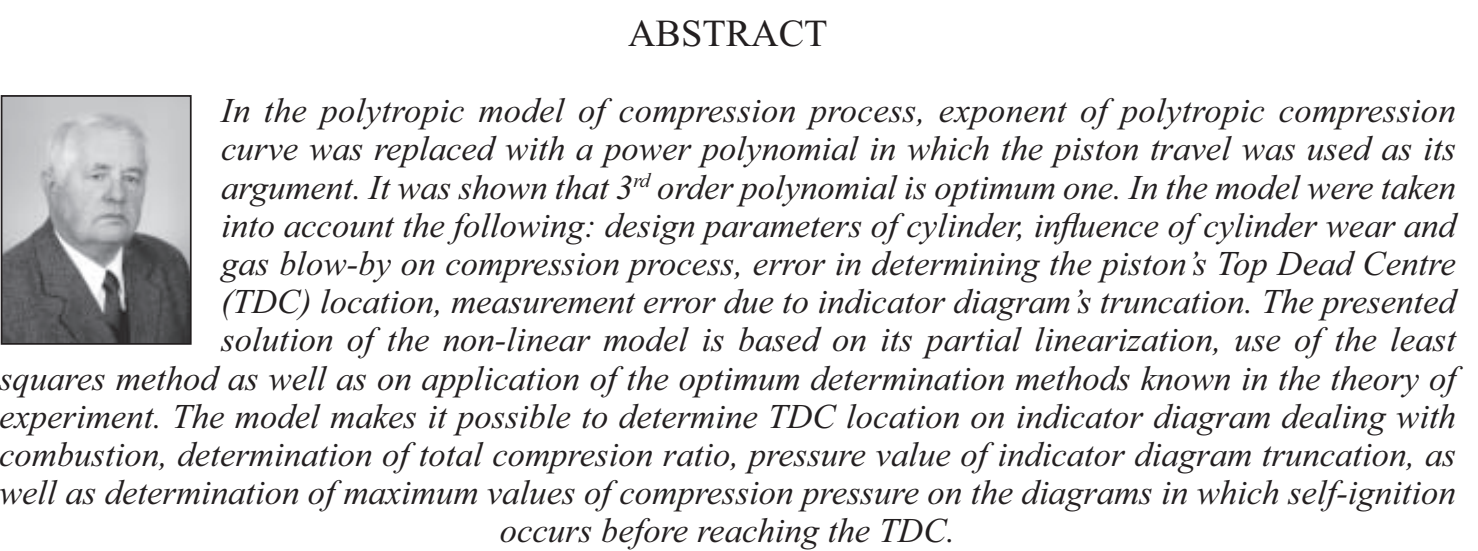

Keywords: Top Dead Centre, compression ratio, ship engine indicator diagram

\section{INTRODUCTION}

Indication of ship engines has been used for regulating and diagnosing purposes since the beginning of their use in shipbuilding. Mechanical indicators used for that purpose make it possible only to determine maximum pressure values of combustion and compression and to roughly assess run of working processes in cylinders on the basis of developed indicator diagrams.

Appearance, in the 1970s, of electronic pressure analyzers often called also MIP (mean indicated pressure) calculators and application of computer technique generally have not changed situation in the area of gaining information from indicator diagrams.

In the presently offered pressure analyzers no important progress has been done as compared with processing capabilities of NK-5 pressure analyzers of Autronica, a Norwegian firm, which have been the first and most commonly applied instruments of the kind in shipbuilding. In many cases, especially as far as portable pressure analyzers are concerned, one can speak about a regress in comparison with their prototype, i.e. NK-5 model. Most of them is not even capable of determining the mean indicated pressure. In any of them no advanced processing of indicator diagrams can be carried out e.g. in the form of analyzing indicator diagram within pressure range as well as determining heat emission characteristics which can serve as a basic source of diagnostic information. Such situation results from met difficulties in indicating ship engines in the domain of crankshaft rotation angle and from lack of mathematical models applicable to pressure analyzers for automatic analyzing the indicator diagrams. However a main abstacle is the difficulty in determining TDC location on indicator diagrams with a sufficient accuracy, i.e. not lower than $\pm 0.3^{\circ} \mathrm{OWK}$ (degree of Crankshaft Rotation Angle). The task is especially difficult in the case of measurements carried out on indicator valves because of deformations of pressure runs, introduced by gas passages and indicator valves themselves.

In the case of low-speed engines when self-ignition occurs after reaching the TDC, peaks of pure compression can be often observed, such as shown in the indicator diagram for RTA52 engine (Fig. 1).

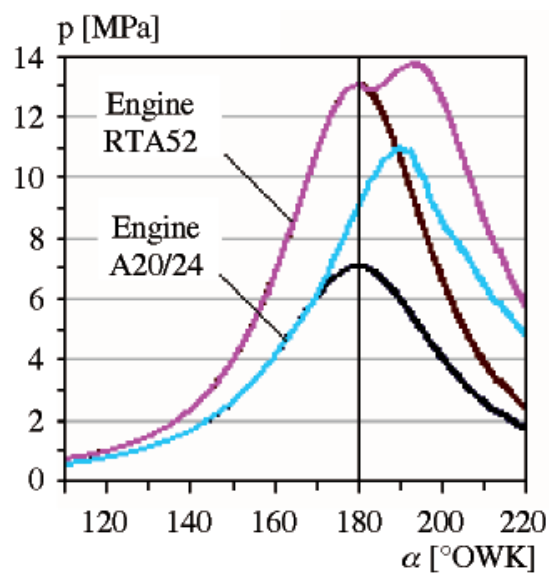

Fig. 1. Typical indicator diagrams of ship engines: - for RTA52 engine of $p=1,8 \mathrm{MPa}, n=137 \mathrm{rpm}$, for Al20/24 engine of $p_{e}=1,8 \mathrm{MPa}, n=750 \mathrm{rpm}$. 
The pure decompression runs on the right from the selfignition points were determined by means of extrapolation technique. In such case one assumes that the piston's TDC occurs in the maximum compression pressure point or the point of the first dervivative's transition through zero. Location of the TDC by using this method may be burdened with large values of errors. Determination of the TDC is connected with specific difficulties when self-ignition occurs just in the TDC or earlier as in the case of medium-speed engines (see Fig. 1 - A20/24 engine). Large difficulties in measuring and analyzing are introduced by the problem of assessment of cylinder tightness, which is usually performed on the basis of maximum compression pressure values. If self-ignition events occur before reaching the TDC then it is necessary to stop delivering the fuel oil to the tested cylinders, that can be allowed in service conditions only in special cases because of possible loss of tightness of fuel system. Large variability of load is often observed, e.g. in electric generating set driving engines, that can make obtaining comparable measurement results of compression pressure in particular cylinders, difficult.

\section{MODEL OF COMPRESSION CURVE APPROXIMATION}

The simplest model of compression process is polytropic one which is usually presented in the following form:

$$
\mathrm{pV}^{\mathrm{m}}=\text { const }=\mathrm{c}
$$

where:

$\mathrm{V}$ - gas volume

$\mathrm{m}$ - exponent of polytropic curve, $\mathrm{m}=$ const.

The maximum compression pressure values applied in contemporary ship engines, exceed $5 \mathrm{MPa}$, and in the case of highly supercharged ones $-10 \mathrm{MPa}$. For this reason the assumption of $\mathrm{m}=$ const will usually lead to excessive errors in determining the thermodynamic parameters [1]. For the highly supercharged engines values of the mentioned errors can even excced $5 \%$.

Having replaced - in the polytropic model - the compression curve exponent $\mathrm{m}=$ const with the exponent $\mathrm{m}_{\mathrm{x}}=$ var, finding the logarithm and differentiating the model (1), one obtains a non-uniform differential equation of the compression process in piston engine, e.g. that in the domain of the piston travel $\mathrm{S}_{\mathrm{x}},(2)$ :

$$
\mathrm{p}^{-1} \frac{\mathrm{dp}}{\mathrm{d} \mathrm{S}_{\mathrm{x}}}+\frac{\mathrm{dm}_{\mathrm{x}}}{\mathrm{d} \mathrm{S}_{\mathrm{x}}} \ln \mathrm{V}+\mathrm{m}_{\mathrm{x}} \mathrm{V}^{-1} \frac{\mathrm{dV}}{\mathrm{d} \mathrm{S}_{\mathrm{x}}}=0
$$

It should be added that the above presented model is that of polytropic process. Its equation does not posess any solutions in the domain of elementary functions. The known solutions of the differential equations of the compression process $[2,3]$ are of a cognitive character only and thus they are not applicable to diagnostic problems. The main limitations are: that TDC location is a priori assumed known, lack of association of the mathematical model with technical state of piston-cylinder system, as well as the not taking into account the disturbances introduced by gas passages, indicator valves and measuring gauges.

If the exponent of compression curve cannot be asssumed constant and form of its function is unknown then it is natural to assume that the exponent $\mathrm{m}_{\mathrm{x}}$ can be approximated by the power polynomial of a- order $[7,8,10$, ], e.g. that in the domain of the piston travel $\mathrm{S}_{\mathrm{x}}$ :

where:

$$
\mathrm{m}_{\mathrm{x}}=\mathrm{m}_{0}+\mathrm{m}_{1} \mathrm{~S}_{\mathrm{x}}+\ldots+\mathrm{m}_{\mathrm{a}} \mathrm{S}_{\mathrm{x}}^{\mathrm{a}}
$$

$\mathrm{m}_{0}, \mathrm{~m}_{1}, \ldots, \mathrm{m}_{\mathrm{a}}-$ polynomial coefficients.
The earlier attempts to express the exponent $m_{x}$ as well as the thermodynamical model directly in the domain of crankshaft rotation angle were abandoned due to a high complicity of the model and associated computational difficulties $[5,9,11]$.

Results of analyses of approximation quality of compression curves by using the least squares method, obtained for ship engines of various types for the values of a within the range of $0 \div 4$ show that the least values of sums of squares of deviations were obtained for $\mathrm{a}=3$ (see Tab. 1) $[7,8,10]$.

Tab. 1. Influence of the order of power polynomial, a, on percentage increase of the sums of squares of deviations as compared with the approximation based on $a=3 ; \alpha$ - approximation interval

\begin{tabular}{|c|c|c|c|c|c|c|c|c|}
\hline \multirow{2}{*}{ No. } & \multirow{2}{*}{$\begin{array}{c}\text { Type } \\
\text { of } \\
\text { engine }\end{array}$} & \multicolumn{5}{|c|}{$\mathbf{a}$} & \multirow{2}{*}{$\begin{array}{c}\boldsymbol{\alpha}_{\mathbf{c}} \\
\text { Number }\end{array}$} & $\begin{array}{c}\text { Number } \\
\text { of } \\
\text { samples }\end{array}$ \\
\cline { 3 - 9 } & $\mathbf{0}$ & $\mathbf{1}$ & $\mathbf{2}$ & $\mathbf{3}$ & $\mathbf{4}$ & & & \\
\hline 1 & 6 AL20/24 & 15900 & 98 & 21 & 0 & 20 & $70-168$ & 981 \\
\hline 2 & $40 \mathrm{DM}$ & 2940 & 840 & 54 & 0 & 54 & $80-175$ & 951 \\
\hline 3 & 5 RTA52 & 13900 & 1340 & 21 & 0 & 21 & $100-175$ & 751 \\
\hline 4 & 6RTA58 & 6820 & 790 & 3 & 0 & 3 & $80-175$ & 951 \\
\hline
\end{tabular}

The above given results justified to assume the $3^{\text {rd }}$ order polynomial to be the model of $m_{x}$ exponent in the domain of piston travel $[8,10]$.

The instantaneous volume of gas within cylinder, V, which appears in Eq. (1), can be expressed as follows:

$$
\mathrm{V}=\mathrm{V}_{\mathrm{s}}-\mathrm{V}_{\mathrm{sx}}+\mathrm{V}_{\varepsilon}+\mathrm{V}_{\mathrm{z}}+\mathrm{V}_{\mathrm{bx}}
$$

where:

$\mathrm{V}_{\mathrm{s}}-$ piston displacement volume,

$\mathrm{V}_{\mathrm{sx}}^{\mathrm{s}}$ - piston volume corrresponding with the piston travel done starting from the BDC (Bottom Dead Centre),

$\mathrm{V}_{\varepsilon}$ - geometrical volume of compression chamber,

$\mathrm{V}_{\mathrm{z}}^{\varepsilon}$ - change of cylinder volume due to wear of elements and influence of assembling process,

$\mathrm{V}_{\mathrm{bx}}$ - apparent change of cylinder volume due to blow-by of gases.

The quantity $\mathrm{V}_{\mathrm{bx}}$ constitutes the volume by which the cylinder volume $\mathrm{V}$ should be increased to obtain changes of gas parameters resulting from gas losses due to blow-by effects. It is a function of piston travel.

By dividing Eq. (4) by the displacement volume $\mathrm{V}$, the particular volumes in Eq. (4) can be transformed to the following dimensionless form [8]:

$$
\mathrm{v}=1-\mathrm{v}_{\mathrm{sx}}+\mathrm{v}_{\varepsilon}+\mathrm{v}_{\mathrm{z}}+\mathrm{v}_{\mathrm{bx}}
$$

The value $\mathrm{v}_{\mathrm{sx}}$ is equal to the dimensionless piston travel $\mathrm{s}_{\mathrm{x}}$ done starting from the BDC, which is expressed by the known formula for central crank system:

$$
\mathrm{S}_{\mathrm{x}}=\frac{1}{2}\left(1-\lambda^{-1}+\sqrt{\lambda^{-2}-\sin ^{2} \alpha}-\cos \alpha\right)
$$

where:

$\alpha-$ craknshaft rotation angle counted from the BDC

$\lambda-$ crank radius/connecting-rod- length ratio

To crank systems of articulated connecting-rods appropriate kinematic relations should be applied.

By introducing the notation:

$$
\mathrm{v}_{\mathrm{xs}}=1-\mathrm{v}_{\mathrm{sx}}=1-\mathrm{s}_{\mathrm{x}}
$$

and

$$
\mathrm{v}_{\mathrm{cx}}=\mathrm{v}_{\varepsilon}+\mathrm{v}_{\mathrm{z}}+\mathrm{v}_{\mathrm{bx}}
$$

the basic model of compression process can be expressed as follows: 


$$
\mathrm{p}\left(\mathrm{v}_{\mathrm{xs}}+\mathrm{v}_{\mathrm{cx}}\right)^{\mathrm{m}_{\mathrm{x}}}=\mathrm{const}=\mathrm{c}
$$

for which - according to the made assumptions - the following is valid:

$$
m_{x}=m_{0}+m_{1} s_{x}+m_{2} s_{x}^{2}+m_{3} s_{x}^{3}
$$

For diagnostic purposes the notion of the total compression ratio $\varepsilon_{\mathrm{c}}$ determinable from the below given expession, is introduced:

$$
\varepsilon_{\mathrm{c}}=\frac{1+\mathrm{v}_{\mathrm{c}}}{\mathrm{v}_{\mathrm{c}}}
$$

where:

$\mathrm{v}_{\mathrm{c}}=\mathrm{v}_{\mathrm{cx}}\left(\mathrm{s}_{\mathrm{x}}=1\right)$

The volume $\mathrm{v}$ characterizes influence of gas blow-by and changes of compresion chamber volume on compresion ratio value.

The notion „total compression ratio" was attributed to the quantity $\varepsilon_{c}$ to distinguish it from the notion of the real compression ratio defined as the ratio of the working space volume at the instant of closing the ports or valves during compression stroke and the compresion chamber volume.

In the performed calculations the simple linear relationship of apparent increase of cylinder volume due to gas blow-by: $\mathrm{v}_{\mathrm{bx}}=0.01 \mathrm{~s}_{\mathrm{x}}$, was assumed. Assumption of another form of the blow-by function would not make calculations more difficult.

It should be mentioned that in service conditions indication process is realized in the domain of piston travel or time. Due to influence of gas passages and indicator valves, locations of the dynamic TDC measured on engine and that thermodynamic determined on indicator diagram can significantly differ to each other. For Sulzer A medium-speed engines the differences reach $3,5^{\circ} \mathrm{OWK}$ at the rotational speed of $750 \mathrm{rpm}[6]$.

For this reason the angle axis of indicator diagram, $\alpha_{\mathrm{I}}$, should be shifted relative to the angle axis of kinematic system, $\alpha$, by the TDC location error $[7,8,10]$, that is expressed as follows:

$$
\alpha=\alpha_{\mathrm{I}}+\Delta \alpha_{\mathrm{G}}
$$

where:

$\Delta \alpha_{\mathrm{G}}-$ the TDC location error.

Value of the TDC location error is searched for

The gauges used for indication of ship engines are generally dynamic ones in which constant component is eliminated. The effect of truncation of indicator diagrams from below occurs, which can be clearly observed on the runs of initial compression process in cylinders of 5RTA52 engine (Fig. 2). In this case measurements were performed by means of a pressure gauge of Kistler firm.

Hence it should be assumed that the real pressure $p$ in the compression curve model (9) is the following sum $[7,8,10]$ :

$$
\mathrm{p}=\mathrm{p}_{\mathrm{I}}+\Delta \mathrm{p}_{\mathrm{I}}
$$

where:

$\mathrm{p}_{\mathrm{I}} \quad$ - recorded pressure run (indicator diagram)

$\Delta \mathrm{p}_{\mathrm{I}}-$ error of indicator diagram truncation (offset).

\section{APPROXIMATION OF COMPRESSION PRESSURE RUN BY MEANS OF GLOBAL MODEL AND DETERMINATION OF VALUES OF PARAMETERS}

The global model of approximation of compression curve constitutes the set of Eqs. (6), (9), (10), (11), (12) i (13).
The input quantities and parameters of the model are the following:

+ the recorded indicated pressure $p_{I}$

+ the crankshaft rotation angle $\alpha,(6)$

+ the design (construction) parameter $\lambda,(6)$.

The searched-for quantities are the following:

1 values of the constants of compression curve exponent $\mathrm{m}_{0} \div \mathrm{m}_{3},(10)$, and of the constant $\mathrm{c}$, (9)

1 value of the piston's TDC location error $\Delta \alpha_{\mathrm{G}},(12)$

1 the total compression ratio $\varepsilon_{c},(11)$

$\lambda$ error (pressure) of indicator curve truncation, $\Delta \mathrm{p}_{\mathrm{I}}$, (13).

To determine the above mentioned parameters was used the least squares approximation method whose essence consists in finding the minimum value of the functional:

where:

$$
\mathrm{Sp}=\operatorname{MIN} \sum_{\mathrm{n}_{1}}^{\mathrm{n}_{\mathrm{r}}}\left(\mathrm{p}_{\mathrm{n}}-\hat{\mathrm{p}}_{\mathrm{mn}}\right)^{2}
$$

$\mathrm{Sp}$ - sum of squares of deviations for approximated run

MIN - operator of minimum

$\mathrm{p}_{\mathrm{n}} \quad$ - corrected indicated pressure

$\hat{\mathrm{p}}_{\mathrm{mn}}$ - pressure obtained from approximation

$\mathrm{n}_{1}$ - sample number at the beginning of the approximation interval $\alpha_{\mathrm{cl}}$

$\mathrm{n}_{\mathrm{r}}$ - sample number at the end of the approximation interval $\alpha_{\mathrm{cr}}$

To determine values of the parameters a linearization of the model (9) was applied, realized by finding the logarithms, twofold application of the least squares criterion (14) as well as by using the method of experiment theory [4], in order to obtain optimum values of the parameters $\Delta \alpha_{\mathrm{G}}, \varepsilon_{\mathrm{c}}$ and $\Delta \mathrm{p}_{\mathrm{I}}$ regarding minimization of the sum of squares expressed by the second criterion $[7,8,10]$.

A number of calculations and analyses was performed for a few types of medium-speed and slow-speed ship engines, from which mutually confirming results were achieved $[7,8$, 10]. The below given presentation of the obtained results is based on the analysis of indicator diagrams of 5RTA52 engine (new one) recorded during sea trials (Fig. 2). $\mathrm{p}[\mathrm{MPa}]$
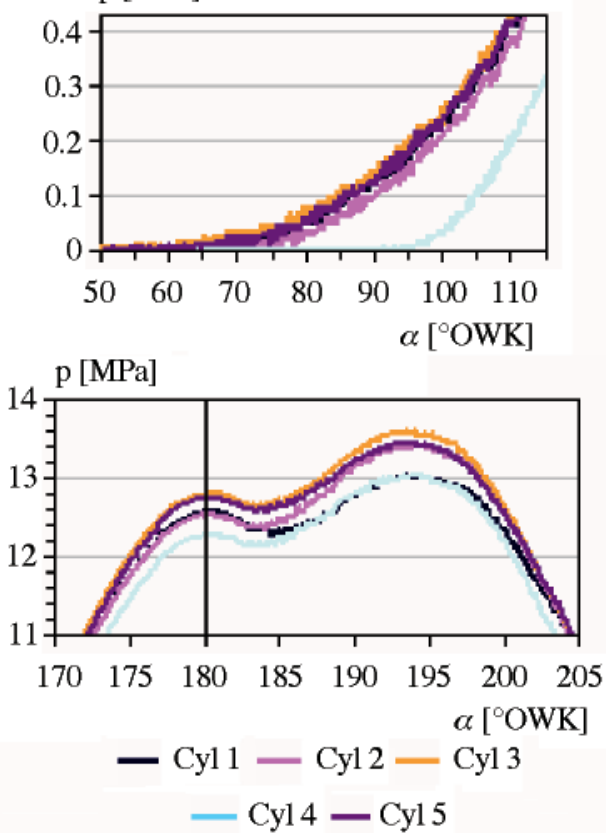

Fig. 2. Runs of compression pressure at the beginning of compression and close to the TDC, for particular cylinders of $5 R T A 52$ engine 
It can be observed (Tab. 2) that the determined values of $\Delta \alpha_{\mathrm{G}}, \varepsilon_{\mathrm{c}}$ and $\Delta \mathrm{p}_{\mathrm{I}}$ for particular cylinders are very close to each other for both options of determination, and also in the case of including two different values of the parameter $\alpha_{\mathrm{cr}}$.

Tab. 2. Results of determination of values of the parameters $\Delta \alpha_{G}, \varepsilon_{c}$ and $\Delta p_{I}$ for cylinders of 5 RTA52 engine (Fig. 2), for $\alpha_{c l}=100^{\circ} \mathrm{OWK}$. option no. 1 - simultaneous determination of $\Delta \alpha_{G}, \varepsilon_{c}$ and $\Delta p_{P}$ option no. 2 - simultaneous determination of $\Delta \alpha_{G}, \varepsilon_{c}$ for the assumed value of $\Delta p_{I}=0,27 \mathrm{MPa}$

\begin{tabular}{|c|c|c|c|c|c|c|}
\hline \multirow{2}{*}{$\begin{array}{c}\text { Option } \\
\text { no. }\end{array}$} & $\boldsymbol{\alpha}_{{ }_{\text {cr }}}$ & \multicolumn{5}{|c|}{$\Delta \boldsymbol{\alpha}_{\mathbf{C}}{ }^{\circ}$ OWK } \\
\cline { 3 - 7 } & $\mathbf{C y l . ~ 1 ~}$ & Cyl. 2 & Cyl. 3 & Cyl. 4 & Cyl. 5 \\
\hline 1 & 180 & -0.35 & -0.25 & -0.25 & -0.25 & -0.50 \\
\hline 1 & 175 & -0.35 & -0.25 & -0.25 & -0.20 & -0.50 \\
\hline 2 & 180 & -0.40 & -0.25 & -0.25 & -0.30 & -0.50 \\
\hline 2 & 175 & -0.35 & -0.25 & -0.25 & -0.20 & 0.50 \\
\hline & & \multicolumn{5}{|c|}{$\boldsymbol{\varepsilon}_{\mathbf{c}}$} \\
\hline 1 & 180 & 13.95 & 14.65 & 14.20 & 13.15 & 14.05 \\
\hline 1 & 175 & 13.95 & 14.80 & 14.30 & 13.10 & 14.00 \\
\hline 2 & 180 & 13.90 & 14.65 & 14.20 & 13.00 & 14.05 \\
\hline 2 & 175 & 13.95 & 14.80 & 14.30 & 13.00 & 14.05 \\
\hline & & \multicolumn{5}{|c|}{$\Delta \mathbf{p}_{\mathbf{I}} \cdot \mathbf{M P a}$} \\
\hline 1 & 180 & 0.225 & 0.270 & 0.275 & 0.260 & 0.265 \\
\hline 1 & 175 & 0.230 & 0.270 & 0.270 & 0.280 & 0.260 \\
\hline
\end{tabular}

The observed differences between values of $\Delta \mathrm{p}_{\mathrm{I}}$ for particular cylinders may awake some doubts (Tab. 2). Results of simulation analysis demostrated that in the case in question the estimation errors of $\Delta \mathrm{p}_{\mathrm{I}}$ values equal to $\pm 20 \%$ lead to the determination errors of $\varepsilon_{c}$ values not greater than $\pm 2 \%$. Therefore it is justified to assume - for calculations - the same $\Delta \mathrm{p}_{\mathrm{I}}$ values for all cylinders, depending on supercharging pressure value.

Analogous results were obtained also from analyses of indicator diagrams for engines of other types $[8,10]$.

\section{DETERMINATION OF THE TDC AND ASSESSMENT OF APPROXIMATION MODEL ADEQUACY}

For analyzing the problem of determination of piston's TDC were used the indicator diagrams where compression pressure maximum was observed (Fig. 2, Fig. 3).

In the case of 6 AL20/24 medium-speed engine the diagrams represented runs of pure compression in tested cylinder (Fig. 3) [8] because self-ignition occurred in the engine before reaching the TDC, under every load.

For measurement performing in cylinder the value of $\Delta \alpha_{G}$ is small and equal to about $0.1^{\circ} \mathrm{OWK}$, but for measuring on indicator valve the value of $\Delta \alpha_{\mathrm{G}}$ is equal to over $-0.9^{\circ} \mathrm{OWK}$ (Fig. 3). It is caused by disturbances from the side of the gas passage and indicator valve itself. It should be mentioned that on the above presented diagrams (Fig. 3) the angular lag between the run on the indicator valve and that in the cylinder could not be observed, though it was equal to $2.7^{\circ} \mathrm{OWK}$.

Identical symptoms as those shown in Fig. 3 take place in the case of slow-speed engines (Fig. 4).

For successive cylinders of 5RTA52 engine the following values of the error $\Delta \alpha_{\mathrm{G}}$ were achieved: -0.35 ; $-0.25 ;-0.25$; $-0.25,-0.5^{\circ} \mathrm{OWK}$. Analyzing indicator diagrams of engines of different types, in some cases one observed $\Delta \alpha_{G}$ values reaching even $1^{\circ} \mathrm{OWK}$. Hence when the TDC location is assumed at the compression pressure maximum point or in the point of transition through zero of its $1^{\text {st }}$-order derivative, one should take into account possibility of making large errors in determining the mean indicated pressure and characteristics of heat emission.
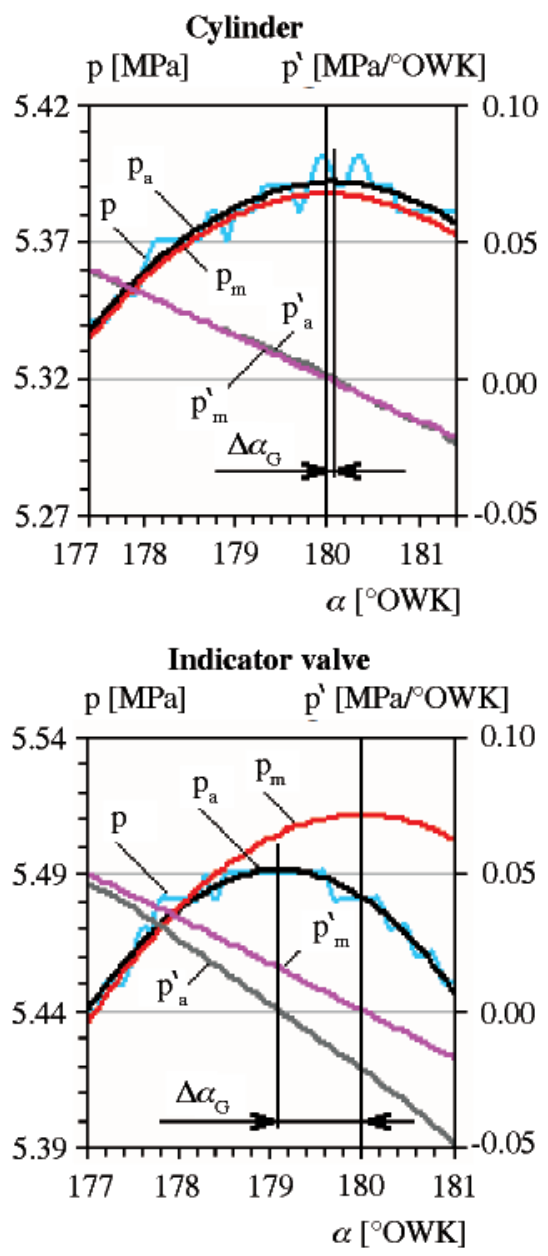

Fig. 3. Comparison of determination results of the TDC location on the runs of pure compression in a cylinder and on indicator valve of 6AL20/24 medium - speed engine $(50 \%$ rated load, $n=750 \mathrm{rpm})$ for the approximation interval $\alpha=100 \div 180^{\circ}$, and obtained by using the approximation model $; p_{a^{\prime}}^{\prime} p_{m}^{\prime}$ - the $1^{\text {st }}$ - order derivatives of the runs in question. The value of $\Delta p_{c}$ was assumed with taking into account supercharging pressure value.

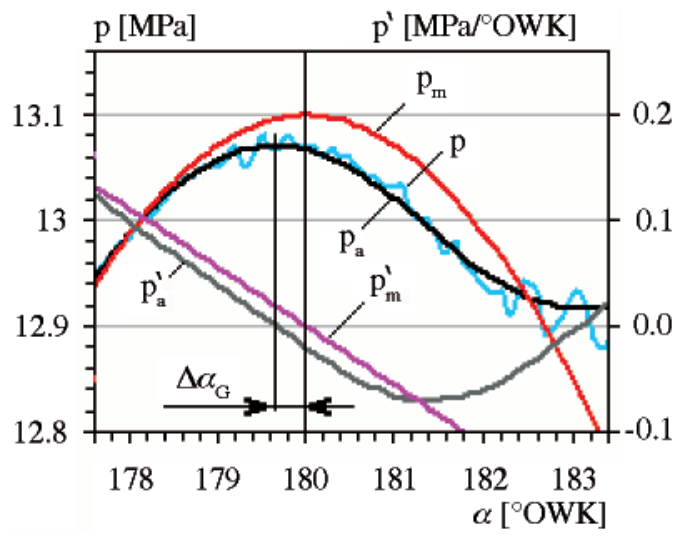

Fig . 4. Comparison of determination results of the TDC location on the indicator diagram of 5 RTA52 slow-speed engine (100\% rated load, $n=130.5 \mathrm{rpm}$, cylinder no.1) for the approximation interval $\alpha_{c}=100 \div 180^{\circ} \mathrm{OWK}$. The value of $\Delta p_{I}=0.27 \mathrm{MPa}$ was assumed with taking into account supercharging pressure value.

The question arises if the TDC locations determined on the basis of approximation by means of the total model are not erroneous and if the method makes it possible to determine the TDC locations in the case when self-ignition occurs before reaching the TDC. For adequacy of the elaborated model speak the results of the TDC determination for e.g. 5RTA52 engine, 
obtained for different values of the ends of the approximation inetrval $\alpha_{\mathrm{cr}} \geq 173{ }^{\circ} \mathrm{OWK}$ (Fig. 5).

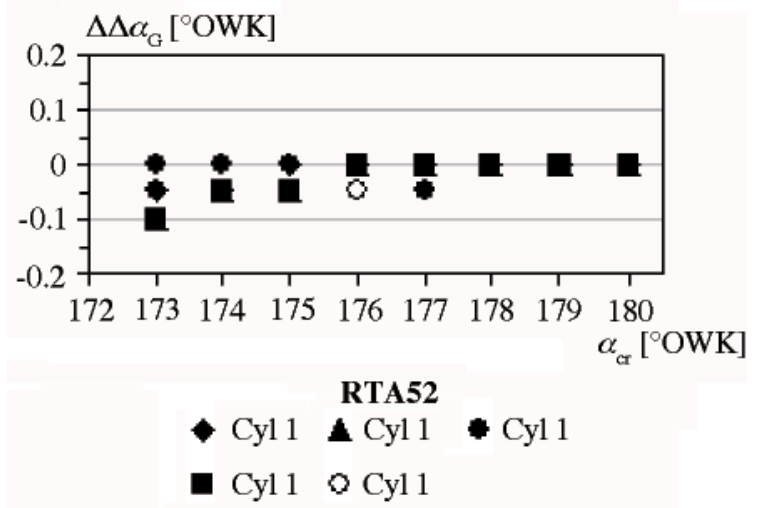

Fig. 5. Influence of end values of the approximation interval $\alpha$ on the increments of error values, $\Delta \Delta \alpha_{G}$ for successive cylinders of $5 R T A 52$ engine, at $\alpha_{c l}=100^{\circ} \mathrm{OWK}$.

The value of $\Delta \Delta \alpha$ did not exceed $0,1^{\circ} \mathrm{OWK}$ at $\alpha=173$ ${ }^{\circ} \mathrm{OWK}$, and at $\alpha_{\mathrm{cr}} \geq 174{ }^{\circ} \mathrm{OWK}$ it did not exceed $0,05^{\mathrm{cr}} \mathrm{OWK}$ (Fig. 5). It means that prevailing influence on value of the sum of squares of deviations has location of the angle axis of the kinematic system relative to the angle axis of the indicator diagram. Identical results were obtained for engines of other types including medium-speed ones $[8,10]$. However for the values $\alpha_{\text {cr }}<165^{\circ} \mathrm{OWK}$ the values of $\Delta \Delta \alpha_{\mathrm{G}}$ can exceed $1^{\circ} \mathrm{OWK}$, which is associated with exceedance of the inflection point (i.e. zero value of the $2^{\text {nd }}$ order derivative) which - in the case of ship engines- is located close to $167^{\circ} \mathrm{OWK}$, counting from the BDC. Correctness of the method in question is confirmed by results of analysis of influence of $\alpha_{c r}$ location on determination errors of maximum compression pressure values when location of the TDC on indicator diagram is known (Fig. 6).

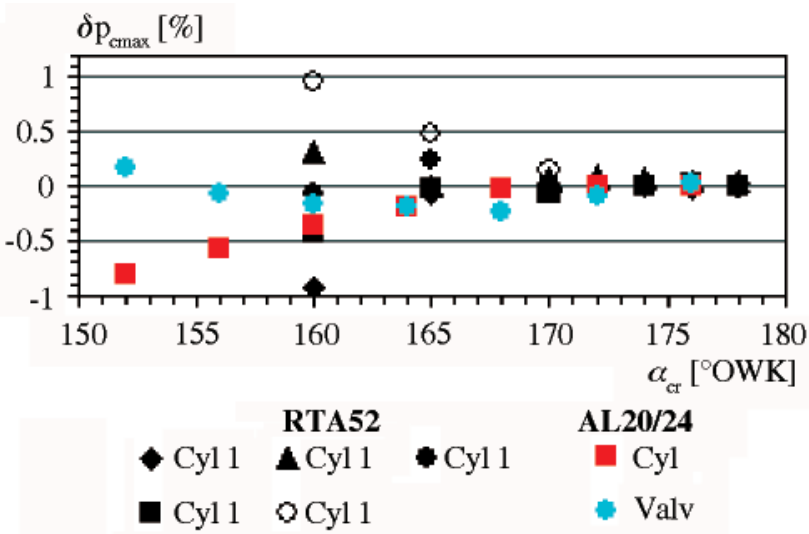

Fig. 6. Influence of value of $\alpha_{c}$ on value of the determination error of maximum compression pressure value, $\delta p$, for successive cylinders of 5 RTA52 engines, as well as for pure compression diagrams of 6AL20/24 engine, at $\alpha_{c l}=100^{\circ} \mathrm{OWK}$

As observed in Fig. 6 the errors of prediction of maximum compression pressure values, $\delta \mathrm{p}_{\mathrm{cmax}}$, do not exceed $1 \%$ even for $\alpha_{\mathrm{cr}}=150{ }^{\circ} \mathrm{OWK}$, and for $\alpha_{\mathrm{cr}}>160{ }^{\circ} \mathrm{OWK}$ they drop to $0,5 \%$ and take very small values for $\alpha_{\text {cr }}>160^{\circ} \mathrm{OWK}$, that confirms adequacy of the elaborated model and the method of determination of parameters.

Appropriate choice of initial value of the approximation interval $\alpha_{c 1}$ greatly influences obtained results $[8,10]$. The values of $\alpha_{\mathrm{cl}}$ should be generally selected in the points where valves or ports are already closed and proper compression is under way and the diagram is only a little disturbed. For ship engines the optimum values of $\alpha$ are contained within the range of $70 \div 100{ }^{\circ} \mathrm{OWK}$ depending on type of such engine $[8,10]$.

\section{ASSESSMENT OF DIAGNOSTIC USEFULNESS OF THE PARAMETER $\varepsilon_{\mathrm{c}}$}

The obtained calculation results showed that for 6AL20/24 engine under various loads, both for measurements performed in cylinder or on indicator valve, the determined values of $\varepsilon_{c}$ are close to each other as compared with the maximum compression pressure values, $\mathrm{p}_{\mathrm{cmax}}$, (Tab. 3).

Tab. 3. Comparison of the values of $\varepsilon$ and $p$ determined on the basis of indicator diagrams of a 6AL20/24 laboratory engine

\begin{tabular}{|c|c|c|c|}
\hline $\begin{array}{c}\text { Load } \\
{[\%]}\end{array}$ & $\begin{array}{c}\text { Place of } \\
\text { measurement }\end{array}$ & $\begin{array}{c}\varepsilon_{\mathrm{c}} \\
{[?]}\end{array}$ & $\begin{array}{c}\mathrm{p}_{\text {cmax }} \\
{[\mathrm{MPa}]}\end{array}$ \\
\hline $\begin{array}{c}50-(\text { pure } \\
\text { compression) }\end{array}$ & Cylinder & 13.9 & 5.28 \\
\cline { 2 - 4 } & Indicator valve & 14.1 & 5.48 \\
\hline \multirow{2}{*}{100} & Cylinder & 14.1 & 7.24 \\
\cline { 2 - 4 } & Indicator valve & 14.3 & 7.38 \\
\hline
\end{tabular}

In the case of the measurement performed on indicator valve the increase of the value of $p_{\text {cmax }}$ by about $35 \%$ resulted in the increase of the value of $\varepsilon_{c}$ by $1,4 \%$ only; and in the case of the measurement in cylinder - the situation was similar. Analyzing the values of $\varepsilon_{c}$ (Tab. 4), determined on the basis of the indicator diagrams of 5RTA52 engine (Fig. 2), one can observe that the value of $\varepsilon_{\mathrm{c}}$ for cylinder no. 4 is significantly smaller, and for cylinder no. 2 - significantly greater than those for the remaining cylinders.

Tab. 4. Comparison of the deviations of values of the parameters $\varepsilon_{c}$ and $p_{\text {cmax }}$ from their mean values, for successive cylinders of $5 R T A 52$ engine: $\delta \varepsilon_{c}$-percentage deviations of $\varepsilon_{c}, \delta p_{\text {cmax }}$ - percentage deviations of $p_{\text {cmax }}$

\begin{tabular}{|c|c|c|c|c|c|}
\hline $\begin{array}{c}\text { Cylinder } \\
\text { number }\end{array}$ & $\mathbf{1}$ & $\mathbf{2}$ & $\mathbf{3}$ & $\mathbf{4}$ & $\mathbf{5}$ \\
\hline$\delta \varepsilon_{\mathrm{c}} \cdot \%$ & -0.7 & 4.6 & 1.4 & -5.7 & 0.4 \\
\hline$\delta \mathrm{p}_{\mathrm{cmax}} \% \%$ & -0.17 & -0.40 & 1.70 & -2.35 & 1.23 \\
\hline
\end{tabular}

If for assessment of tightness of cylinders of 5RTA52 engine one takes into account the values of $\mathrm{p}_{\text {cmax }}$ (Fig. 2) then in the case of cylinder no. 4 the value of $\delta p_{\text {cmax }}=-2.35 \%$ makes it possible to consider the cylinder as that having correct parameters of compression process in spite of that for the cylinder: $\delta \varepsilon_{\mathrm{c}}=-5.7$ $\%$. In the case of cylinder no. 2 attention should be focused on the small value of $\delta \mathrm{p}_{\mathrm{cmax}}=-0.40 \%$, whereas $\delta \varepsilon_{\mathrm{c}}=4.6 \%$, having opposite sign against that of $\delta \mathrm{p}_{\mathrm{cmax}}$. In the case of cylinder no. 4 a setting error was made and the valve was closed much later than in the remaining cylinders, which can be stated from the pressure run occurring at the beginning of compression process (Fig. 2). It is also possible to demonstrate that the phenomenon was not caused by a greater value of the error $\Delta \mathrm{p}_{\mathrm{I}}$ observed in that case $[8,10]$.

It should be also observed that the error $\Delta \alpha_{\mathrm{G}}$ greatly influences the error in determining $\varepsilon_{\mathrm{c}}$ (Tab. 5).

Tab. 5. Influence of the error $\Delta \alpha_{G}$ on the error $\delta \varepsilon_{c}$ in the case of RTA52 engine (for cylinder no. 5)

\begin{tabular}{|c|c|c|c|c|c|c|c|}
\hline $\begin{array}{c}\Delta \boldsymbol{\alpha}_{\mathrm{G}}, \\
{ }^{\circ} \mathbf{O W K}\end{array}$ & -0.3 & -0.2 & -0.1 & 0 & 0.1 & 0.2 & 0.3 \\
\hline $\boldsymbol{\delta} \boldsymbol{\varepsilon}_{\mathbf{c}}, \boldsymbol{\%}$ & -10.9 & -7.1 & -3.4 & 0 & 3.3 & 6.5 & 9.5 \\
\hline
\end{tabular}

In view of the so great influence of the error $\Delta \alpha_{G}$ on the determination error of total compression ratio, $\delta \varepsilon_{c}$, to accurately determine the TDC location becomes especially 
important. In the case of slow-speed engines if measurements are performed carefully both in the aspect of pressure and angle axis measurements, to determine the TDC locations burdened with the error values below $\pm 0.05^{\circ} \mathrm{OWK}$, will be possible.

\section{CONCLUSIONS}

The presented model and method of approximation of indicator diagrams in the compression range make it possible to determine the TDC's location on indicator diagrams also in the case if self-ignition occurs before reaching the TDC.

The so determined TDC is equivalent to that kinematic.

The TDC's location considered identical with the location of maximum value of compression pressure or with zero point of $1^{\text {st }}$ order derivative of pressure run curve is burdened with the error whose value can range from a few decimal parts to $1^{\circ} \mathrm{OWK}$.

The presented method makes it possible to determine the total compression ratio $\varepsilon$. This parameter is more suitable for assessing cylinder tightness losses than maximum value of compression pressure in view of a weak dependence of $\varepsilon_{\mathrm{c}}$ values on engine load level.

- Accuracy of determination of values of the parameter $\varepsilon$, is greatly influenced by value of the error of the TDC's location. Even the values of the error $\Delta \alpha_{\mathrm{G}}$ equal to $\pm 0.3^{\circ} \mathrm{OWK}$ will produce the errors in determining $\varepsilon_{\mathrm{c}}$ values, of the order of $\pm 10 \%$.

In the case of slow-speed engines, on the basis of the approximation model it can be also possible to determine values of the indicator diagram truncation pressure $\Delta \mathrm{p}_{\mathrm{I}}$. However it seems that in the case of service measurements it would be better to resign from determination of value of the parameter in question and to assume - for calculations - its value by taking into account supercharging pressure value.

\section{BIBLIOGRAPHY}

1. Baehr H.D.: Thermodynamik. Eine Einführung in die Grundlagen und ihre technischen Anwendungen. Springer, 1973 (edition in Russian, 1977)

2. Benson R.S., Whitehouse N. D.: Internal combustion engines. Pergamon Press, 1979

3. Cichy M.: Calculation of compression and decompression processes by means of the finite difference method (in Polish). Scientific Bulletin of Gdańsk University of Technology (Zeszyty Naukowe Politechniki Gdańskiej), Mechanics (Mechanika), no. 73, Gdańsk 1996

4. Hartman K., Lezki E., Shäfer W.: Statistische Versuchplanung und-auswertung in der Stoffwirtschaft. VEB, Leipzig, 1974 (edition in Russian,1977)
5. Polanowski S.: Errors arising from relating the piston TDC to zero points of derivatives of ship engine cylinder pressure function (in Polish, abstract in English). Proceedings of KONES Conference 2000, (saved in Warsaw Institute of Aeronautics)

6. Polanowski S.: Main sources of measurement errors of mean indicated pressure of ship engines in service conditions (in Polish, abstract in English). Journal of KONES, 1995, (saved in Warsaw Institute of Aeronautics) Polanowski S., Errors in Toking MIP Measurements by Means of Microcomputer Combustion Pressure Analyzer, Journal of Polish CIMAC, Warsaw, 1992.

7. Polanowski S.: Gaining diagnostic information from indicator diagrams of ship engines by using advanced methods of data processing (in Polish, abstract in English). Scientific Bulletin of Polish Naval University, no. 162 K/2, Gdynia 2005

8. Polanowski S.: A study of analysis methods of indicator diagrams in the aspect of diagnostics of ship engines (in Polish). Scientific Bulletin of Polish Naval University, no. 169 A, Gdynia 2007

9. Polanowski S.: TDC determination on combustion indicator diagram. Journal of KONES, 1999, Vol. 6, No 1-2, (saved in Warsaw Institute of Aeronautics)

10.Polanowski S.: Wieloparametrowy model przebiegu ciśnienia sprężania w cylindrze silnika okrętowego. Scientific booklet Akademii Marynarki Wojennej, 1 (160), Gdynia 2005 (abstract in English)

11.Polanowski S.: Wyznaczanie wykładników politropy sprężania dla pozyskania informacji diagnostycznej z wykresu indykatorowego silnika okrętowego. Conference materials KONES 2001.

\section{NOMENCLATURE}

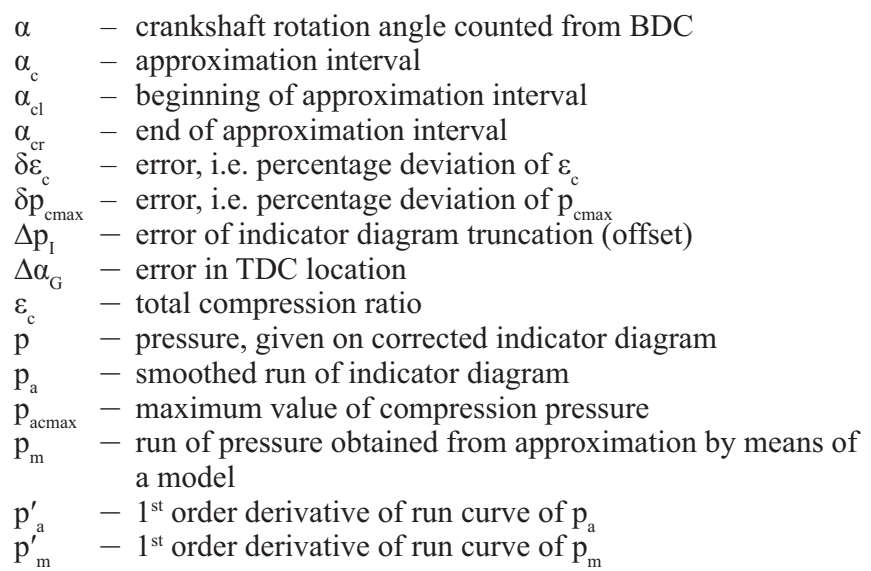

\section{CONTACT WITH THE AUTHOR}

Prof. Stanisław Polanowski

Mechanic-Electric Faculty, Polish Naval University Śmidowicza 69 81-103 Gdynia, POLAND e-mail: SPolanowski@o2.pl 